\title{
Left ventricular assist devices as a part of heart transplant program: Slovakian point of view
}

\section{Eva Goncalvesová* \\ National Cardiovascular Insti- tute, Bratislava, Slovakia}

\author{
KEYWORDS: left ventricular assist device, heart transplantation, advanced heart failure \\ CITATION: Cardiol Croat. 2014;9(9-10):436.
}

*ADDRESS FOR CORRESPONDENCE: National Cardiovascular Institute, PKH 1, 83348 Bratislava, Slovakia Phone: +421 259320263 / E-mail: eva.goncalvesova@nusch.sk

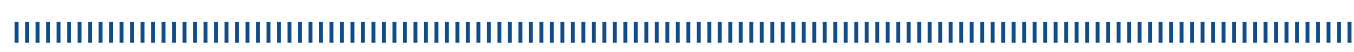

Growing population of advanced heart failure (HF) patients represents one of the major burdens for health care system. Heart transplantation (HTx) is the gold standard for a severe HF treatment but contraindications and lack of donors are ultimate hurdles for its widespread use. Heart replacement using durable mechanical circulatory support, dominantly continuous-flow left ventricular assist devices (LVAD), has grown fast in recent years. It is reasonable to assume that the number of LVAD implantations will continue to grow and will soon exceed the number of HTx. In its recent Guidelines for the diagnosis and treatment of acute and chronic heart failure, European Society of Cardiology recognized a growing role of LVAD in the treatment of terminal HF in selected group of patients. According the Guidelines a LVAD implantation is recommended in selected patients with end-stage HF despite optimal pharmacological and device treatment who are otherwise suitable for heart transplantation, to improve symptoms and reduce the risk of HF hospitalization for worsening HF and to reduce the risk of premature death while awaiting transplantation (class I, level of evidence B). An LVAD should be considered in highly selected patients who have end-stage HF despite optimal pharmacological and device therapy and who are not suitable for heart transplantation, but are expected to survive longer than 1 year with good functional status, to improve symptoms, and reduce the risk of HF hospitalization and of premature death (class IIa, level of evidence B)1.

Heart transplant program started in Slovakia in 1998 (up until then all patient were operated in Prague where the first HTx was performed in 1984). Two hundred twenty two HTx were performed in Slovakia by the end of 2013. One year posttransplant survival was 82, five and ten year survival was 72 and 65 years, respectively. The major problem is lack of donors, only 2.8 heart donors per million population were identified during 2013. A way today how to cope with donor shortage are implantable left ventricular assist devices (LVAD). First durable LVAD (HeartMate II) was implanted in 2006 and by the end of 201351 LVAD's have been implanted in patient who were considered heart transplant candidates (31 as bridge to transplant and 20 as bridge to candidacy). One year total survival was $65 \%, 30$ day mortality after implantation was $24 \%$. Left ventricular assist devices are seen to be a viable way to partially solve heart donors shortage. As a consequence of this situation we offer LVAD implantation to all suitable INTERMACS I - IV heart transplant candidates, ${ }^{2,3}$.

\section{RECEIVED:}

September 22, 2014
LITERATURE IIIIIIIIIIIIIIIIIIIIIIIIIIIIIIIIIIIIIIIIIIIIIIIIIIIIIIIIIIIIIIIIIIIIIIIIIIIIIIIIIIIIIIIIIIIIIIIIIIIIII

1. McMurray JV, Adamopoulos S, Anker SD, et al; ESC Committee for Practice Guidelines. ESC Guidelines for the diagnosis and treatment of acute and chronic heart failure 2012: The Task Force for the Diagnosis and Treatment of Acute and Chronic Heart Failure 2012 of the European Society of Cardiology. Developed in collaboration with the Heart Failure Association (HFA) of the ESC. Eur Heart J. 2012;33(14):1787-847.

2. Goncalvesova E, Hulman M, Olejarova I, et al. Initial experience with an implantable left ventricular assist device in the heart transplant programme of the National Institute of Cardiovascular Diseases. Cardiol. 2008;17(3):101-8.

3. Goncalvesová E. Durable left ventricular assist devices - the minimum for referring cardiologists. Cor et Vasa. 2013;55(4):e377-e382. 